\title{
Voxcom: A system for analyzing natural speech in real time
}

\author{
MURRAY ALPERT \\ New York University Medical Center, New York, New York \\ FRANK MEREWETHER \\ Haskins Laboratories, New Haven, Connecticut \\ PETER HOMEL \\ New York University Medical Center, New York, New York \\ JACK MARTZ \\ Brooklyn VA Hospital, Brooklyn, New York \\ and \\ MORT LOMASK \\ Buxco Electronics, Sharon, Connecticut
}

\begin{abstract}
A system is described for analyzing recorded natural speech in real time using a microcomputer. Recordings up to 15 min in length can be analyzed in terms of fundamental frequency, amplitude, length of utterances, and pauses. Although primarily developed for clinical research, the system has applicability to other research areas involving speech.
\end{abstract}

We have been developing a computer system for analyzing recorded samples of natural speech in real time. Our interest in this system centers on using it to examine acoustic correlates of various neuropsychiatric disturbances. However, since the system provides information about such basic speech variables as fundamental frequency (pitch), amplitude (loudness), and time duration of utterances and pauses, the system is readily applicable to a number of other research situations that involve the measurement of these prosodic features.

\section{THE PHYSICAL SYSTEM}

Figure 1 shows the general physical layout of the Voxcom system, which consists of three main components: a stereo cassette tape deck (Nakamichi LX-3), a microcomputer equipped with an analog-to-digital (A/D) conversion system (Northstar Horizon with a Tecmar TMAD212 Analog to Digital), and a multifunction analog signal processing unit designed by Buxco Electronics. The role of the Buxco unit will be made clear in the discussion below. Generally, however, it provides the circuitry for filtering and transforming the signals that go from the tape deck to the A/D board of the Northstar microcomputer.

This work was supported in part by USPHS Grants No. MH35976 and MH37952. The first author's address is: Millhauser Laboratories, 3rd floor, New York University Medical Center, 550 First Avenue, New York, NY 10016

\section{INITIAL SIGNAL PROCESSING}

The system is designed to analyze recorded samples of natural speech obtained during the course of psychiatric interviews. Patient and interviewer have their own individual unidirectional microphones so that they are recorded on separate channels of the tape deck. In the case of the patient, a head microphone is used in order to keep the distance between patient and microphone constant.

Signals from the interviewer's and patient's channels are initially processed by the Buxco unit before they undergo analysis by the computer. As can be seen in Figure 1, the interviewer's signal is sent to a circuit in the Buxco unit which detects input voltages above an adjustable threshold. When the input exceeds threshold, the circuit then outputs a $+5 \mathrm{~V}$ TTL signal to the parallel port of the Northstar for the duration that the input signal is above threshold. Output from the circuit goes to $0 \mathrm{~V}$ when the input from the interviewer's channel goes below threshold. During the course of data collection, analysis of the patient's channel is interrupted whenever the interviewer speaks (i.e., whenever a TTL signal is detected at the parallel port).

The patient's signal passes through a different series of circuits. The raw ac signal that comes from the tape deck is first passed through a high-pass/low-pass band filter in order to restrict the frequencies in that signal to a range around the speaker's fundamental frequency. The range between the filters can be adjusted for particular samples of speech; for example, it is usually set between 


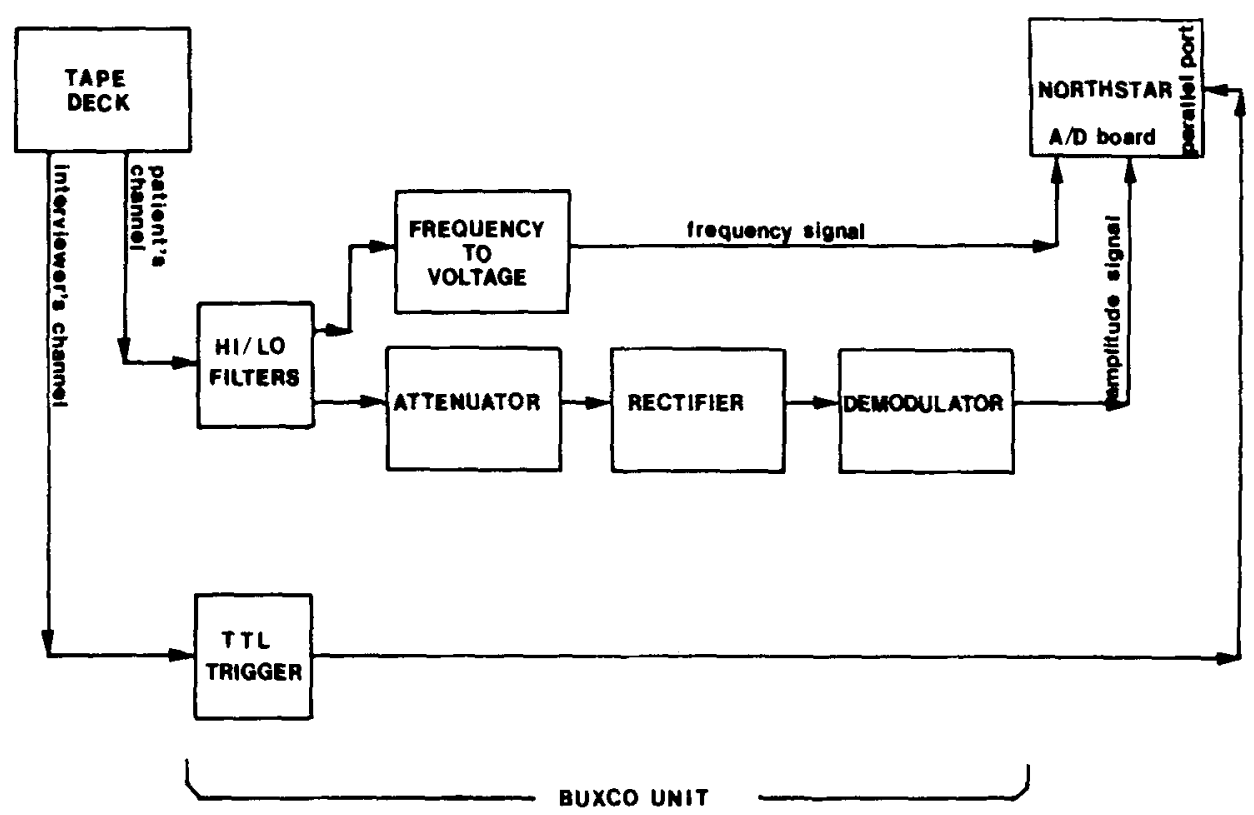

Figure 1. Physical layout of the Voxcom system.

80 and $140 \mathrm{~Hz}$ for male voices and between 120 and $280 \mathrm{~Hz}$ for female voices.

Once filtered, the patient's signal is then split into two parallel lines, one of which is analyzed for frequency information and the other for amplitude information. The frequency signal passes through a frequency-to-voltage converter, with every $50-\mathrm{Hz}$ increment of input signal being equal to a $1-\mathrm{V}$ increment in output. This signal is then sent to one of the channels on the A/D converter board with a resolution of 200 counts per volt. The signal on the amplitude line is fed to an amplifier with a stepped attenuator, then is full-wave rectified to a dc signal and finally demodulated to produce a smooth signal roughly equivalent to spoken syllables. This signal then goes to another channel on the $A / D$ converter board.

\section{THE VOXCOM SOFTWARE}

\section{Data Collection}

Figure 2 shows two examples of how the amplitude signal would look if one were to do an oscilloscopic tracing of the signal at the point of its input into the A/D converter in the Northstar microcomputer. The variation in voltage across time corresponds to the changes in loudness or stress produced by the patient when speaking.

We define an utterance as being marked by an amplitude which is above some threshold of background noise for $100 \mathrm{msec}$ or more, a gap as being marked by an amplitude that is below threshold for at least $200 \mathrm{msec}$, and a peak as a point of maximum amplitude relative to the values of amplitude immediately preceding and following that point.

The Voxcom software is designed to measure the following features pertaining to utterances, gaps, and peaks: (1) number of utterances, gaps, and peaks; (2) mean and variance of the time durations of utterances, gaps, and peaks (with a resolution of $10 \mathrm{msec}$ ); (3) mean and variance of the log of the amplitudes of peaks, as well as of the frequencies corresponding to those peaks; (4) the correlation between peak amplitude and peak frequency; (5) the distribution of peaks within utterances (i.e., how many 1-peak utterances, 2-peak utterances, etc., were there), as well as summary information about the duration of the peaks in those utterances.

The arrangement of the data collection routine is given in Figure 3. Prior to the initiation of data collection, the threshold for background noise (i.e., nonspeech sounds) is set, either by arbitrary rule or by sampling a section of the recording at a time when the patient is not speaking. Upon the start of data collection, the voltages at the amplitude and frequency channels are sampled every $10 \mathrm{msec}$, based upon the real-time clock provided on the A/D board. The program waits until the first rise in amplitude occurs (i.e., above the preestablished threshold). As the sampling continues, the values of amplitude and frequency are stored and each succeeding value of the amplitude is compared with the previous one in order to determine whether the amplitude is increasing or is showing a decrease.

If the amplitude shows a decrease, the routine determines whether a peak has occurred. This is done by comparing the obtained change across time with the parameter peak min (peak minimum height), which defines the minimum amount of decrement in amplitude across time for a peak. If the change is more than pkminh (defined below), the routine stores the value of the amplitude at the peak as well as the reading in the frequency channel and the value of the clock coincident with the peak. If the change in amplitude is greater than pkminh and the amplitude begins to rise again before declining 

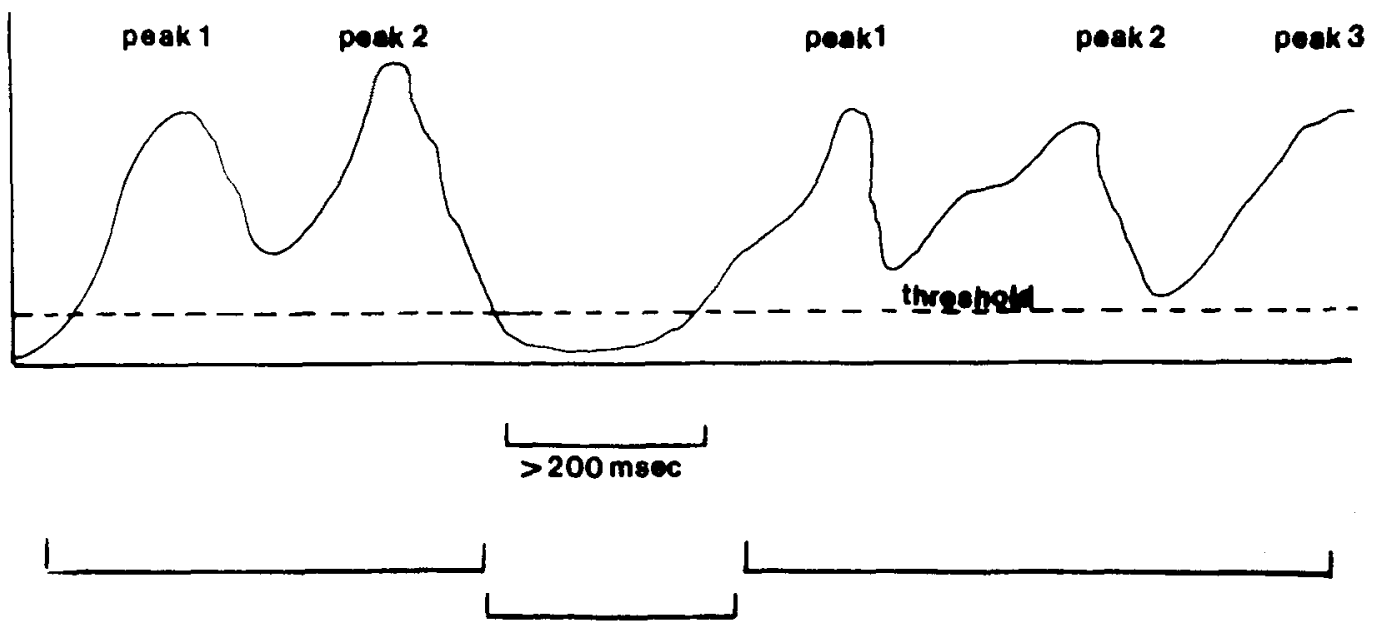

$\begin{array}{lll}\text { UTTERANCE } 1 & \text { GAP } & \text { UTTERANCE } 2\end{array}$

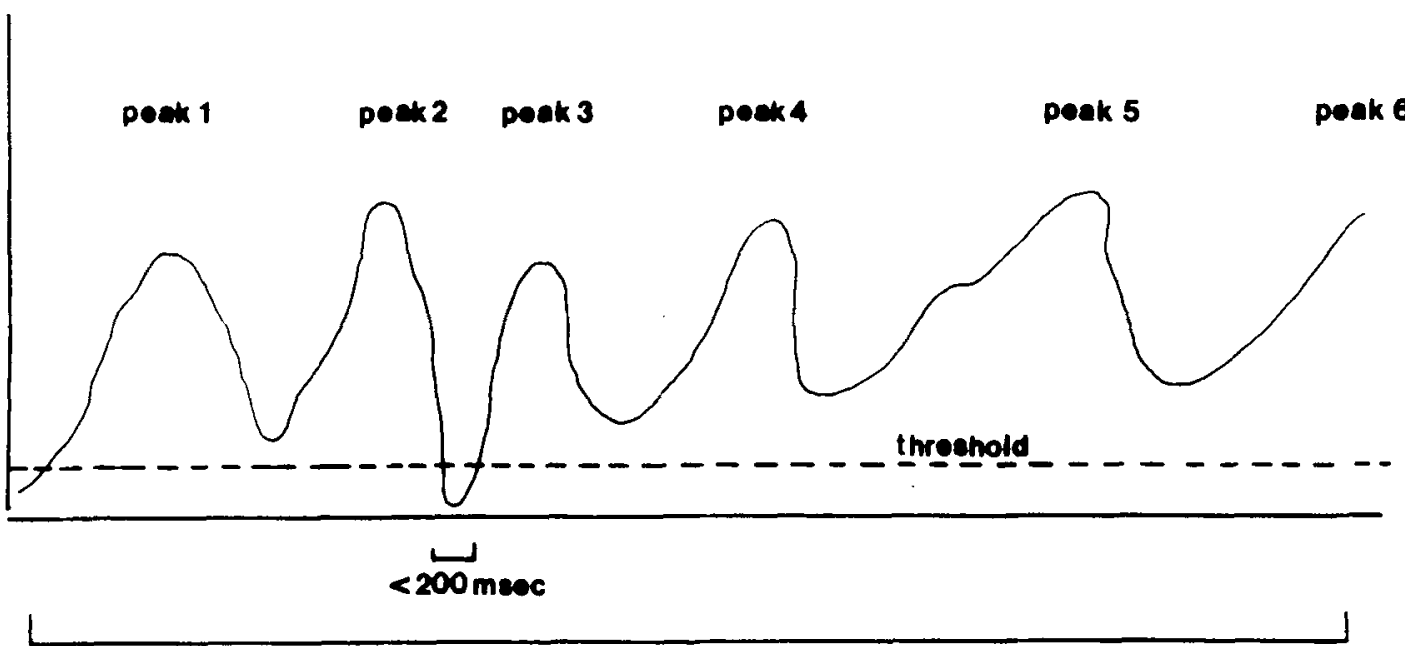

\section{UTTERANCE}

Figure 2. Hypothetical oscilloscopic tracings of two samples of speech to illustrate how Voxcom detects a gap between utterances.

to below threshold, then the process of sampling and comparing to find the next peak goes on as it did before. The portion of the upper panel in Figure 2 labeled utterance 1 illustrates an utterance consisting of two peaks which succeed one another without any decline below threshold. Similarly, the portion of the upper panel labeled utterance 2 illustrates an utterance consisting of at least three peaks which succeed one another without any decline below threshold.

If the decline in amplitude does go below threshold at any point and (1) it remains that way for $200 \mathrm{msec}$ or longer and (2) the preceding activity in the amplitude channel was above threshold for $100 \mathrm{msec}$ or longer, then a gap ending an utterance is detected and the information pertaining to the utterance (duration of utterance, number of peaks in the utterance, and amplitudes, frequencies, and time durations of each peak) is stored in another data array. This is shown in the center portion of the upper panel in Figure 2. The routine will then clock the du- ration of "quiet" while waiting for the signal to rise, indicating the beginning of another utterance. A decline below threshold that lasts less than $200 \mathrm{msec}$ does not count as a gap, and so the following peak is counted as belonging to the same utterance as the peak which occurred before that decline. An illustration of this can be seen in the lower panel of Figure 2.

\section{Other Software Features: \\ Parameters and Toggles}

The data collection routine depends upon several parameters which can be altered from the computer console. These parameters include (1) uttmin and gapminminimum time duration for detecting whether an utterance or gap has occurred; (2) pkminh-the minimum decrement in amplitude needed for a peak to be detected; (3) thresh-the threshold or minimum amplitude that is considered "signal" by the peak detection routine; (4) fmin and fmax-the minimum and maximum values 


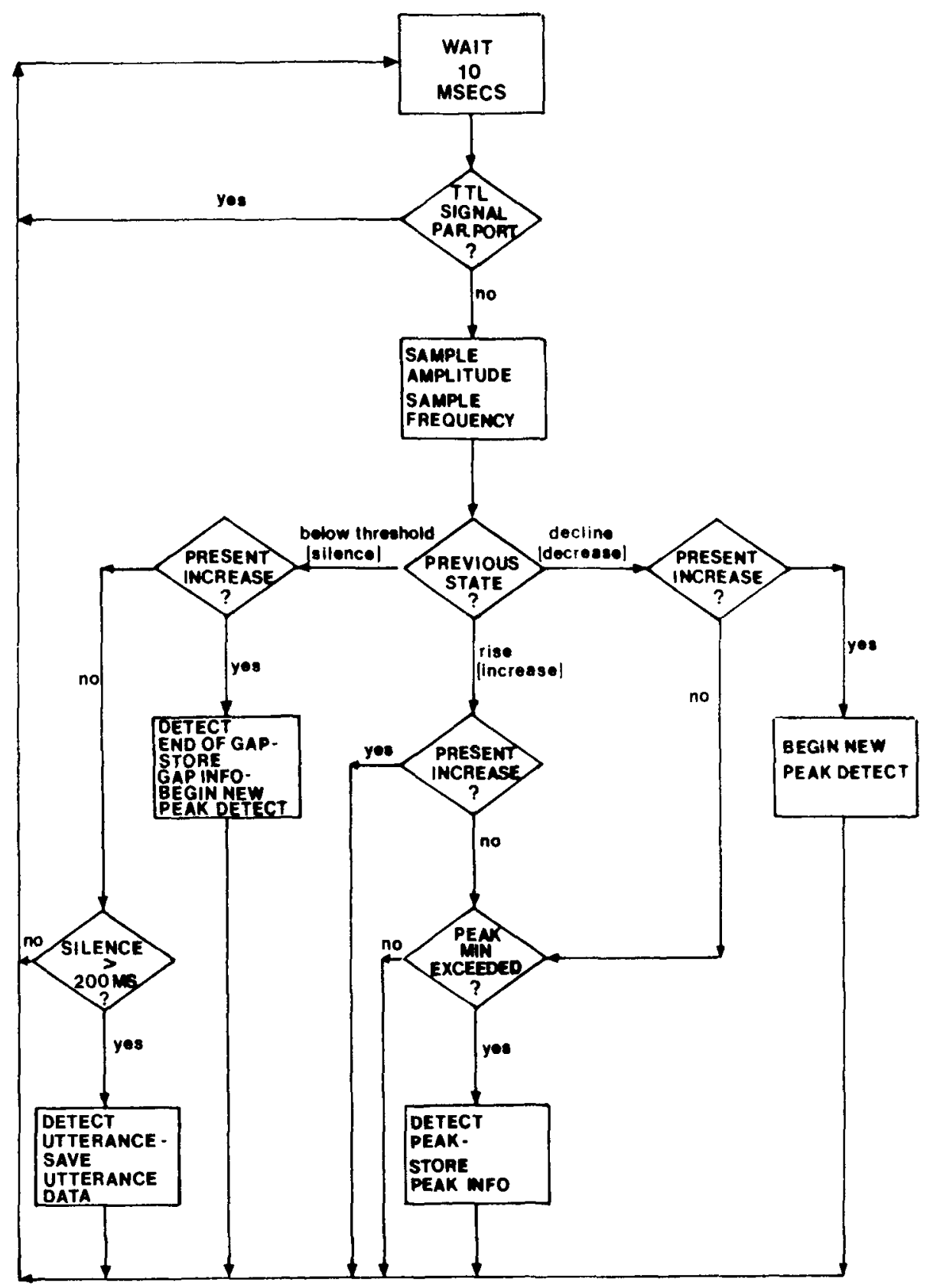

Figure 3. Flow diagram of data Voxcom collection routine.

of peak frequency that are accepted during data collection. The high/low filters of the Buxco unit provide a rolloff of $6 \mathrm{~dB}$ and are designed to reduce harmonics. The parameters fmin and fmax allow for finer control of the range of peak frequencies that are accepted as being "valid" as values of fundamental frequency and are set to about an octave above and below the patient's modular fundamental.

The program also allows the user to set the threshold automatically. A software toggle switch controlled from the computer console engages a routine which measures the average amplitude in the patient's channel and sets the threshold to that level. Normally, one would play a section of the interview when only the interviewer is speaking in order to set the threshold to what should be the loudest source of "noise" in the patient's channel. An additional routine, also under toggle control, is available for registering a calibration signal for both amplitude and frequency. A calibrator produced by Buxco Electronics permits a signal of fixed amplitude and frequency to be recorded on the tape at the same gain level that was used to record the patient. This allows the user to compare obtained values of amplitude and frequency in relationship to signals with known characteristics.

Two routines account for "editing" or temporarily halting data collection. One operates automatically; the other 
is controlled from the console. During the course of each sampling cycle, the parallel port is tested for the presence of a $+5 \mathrm{~V}$ TTL signal from the Buxco unit. As explained above, the TTL signal is sent to the computer whenever an input voltage above a particular threshold level is detected from the interviewer's channel. If the TTL signal is present, data collection is suspended until the signal returns to $0 \mathrm{~V}$. This effectively edits out the interviewer as a whole from the analysis. This "auto-edit" scheme is useful in instances where the interviewer's voice has been picked up by the patient's microphone and is registering above the usual threshold on the patient's channel. This also prevents the analysis routine from including those intervals when the interviewer is talking (and the patient is listening) as gaps in the patient's ongoing speech.

Data collection can also be temporarily halted by engaging a software toggle from the console keyboard. This manual method of editing is useful in cases where extraneous noise (e.g., banging on table, movement of chairs) is picked up by the patient's microphone.

\section{Output of the Analysis Routine}

Table 1 gives an example of the usual output one would obtain from analyzing a portion of a patient's interview using the Voxcom system. A listing of the parameters is given at the beginning. Note that the parameters relating to the calibration values for amplitude and frequency (i.e.,

Table 1

Output of Voxcom Analysis Routine

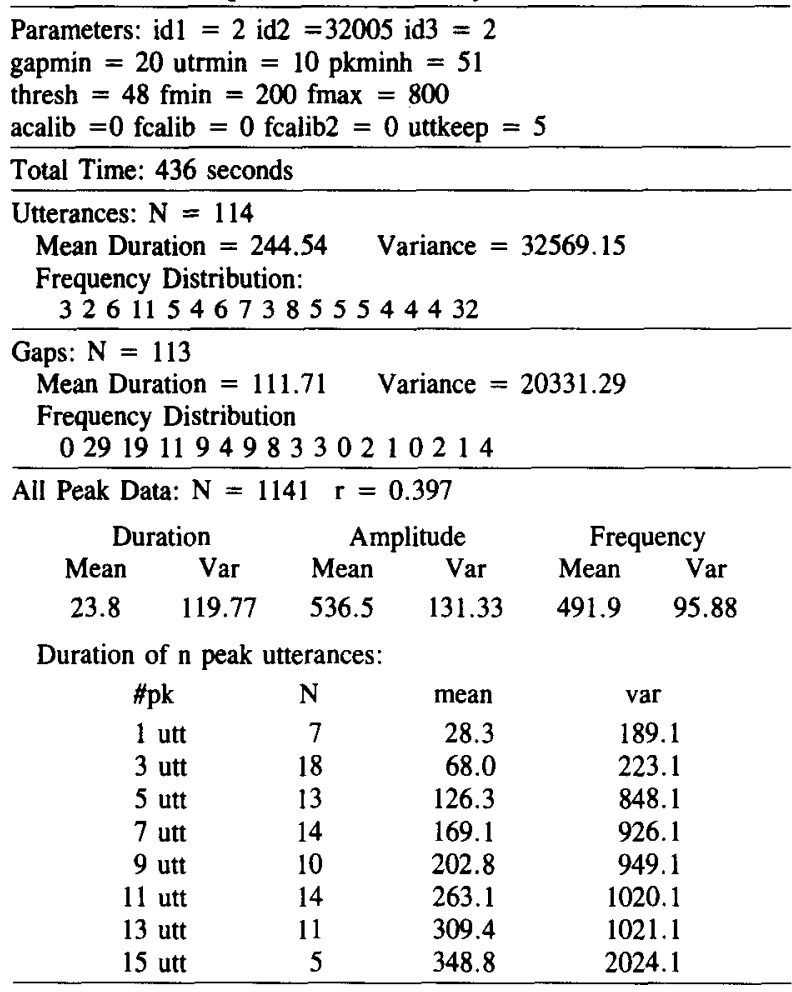

acalib, fcalib, fcalib2) are all equal to 0 , which indicates that this analysis was done without a prior calibration signal. Accordingly, the values pertaining to amplitude and frequency are relative to the original incoming signals measured at the $\mathrm{A} / \mathrm{D}$ channels rather than in terms of $a b$ solute decibels and hertz.

The rest of the output is divided into the following sections: (1) Total seconds: the total time of data collection in seconds. (2) Utterances: this section gives information about the number of utterances, mean duration of utterances in centiseconds (csec), the variance of those durations, and a frequency distribution of the utterance durations (intervals are in terms of 20-csec increments). (3) Gaps: similar to utterances; $n$ of gaps, mean and variance of duration, and frequency distribution. (4) All Peaks: $n$ of all peaks detected; the correlation between the amplitude and frequency of the peaks; the mean duration of the peaks in centiseconds and the variance of their duration; the mean amplitude and the variance of amplitude among all the peaks; mean frequency and variance of peak frequency; and a summary of the "odd" peak utterances, given the number of occurrences of each odd peaked utterance and the mean duration and variance of duration for each set of odd peaked utterances.

A few points of explanation are necessary. First, the use of centiseconds as a unit of time is a matter of scaling convenience, because it yields values that are neither excessively large nor small. In addition, the summary entries for odd peak utterances include both the odd peak data indicated and data for the $n-1$ even peak utterances (e.g., the summary for the 3-peak utterances also includes the data for the 2-peak utterances).

\section{PRESENT AND FUTURE APPLICATIONS OF THE SYSTEM}

At present we are using the Voxcom system to provide objective and quantitative characterizations of the speech patterns found in conditions where there is a disturbance in the ability to express emotion or affect, such as schizophrenia, depression, and other clinically interesting conditions. This study is a follow-up of some earlier work done by our group (summarized in Alpert, 1982) which indicated that one of the acoustic cues that may differentiate some of these clinical groups may be their overall variance in terms of frequency and amplitude: flataffect schizophrenics seem to show less variation in frequency and amplitude than depressives. Another study is under way to identify unique patterns of prosody in the following clinical groups: patients with right hemisphere brain damage; patients with Parkinson's disease; patients with hypertension; and depressive patients. We are particularly interested in the way different types of emotion (e.g., happiness, sadness, anger) are encoded in each of these groups. With some slight modifications in the software, we are also examining the usefulness of a program 
that gives sequential information about peak patterns within utterances, in contrast to the present one which gives nonsequential summary data for the entire sample of speech analyzed.

In conclusion, we feel that the Voxcom system offers a reliable means for doing real-time acoustic analysis of human speech. Its features make it suitable for a number of applications involving voice data which are not limited to clinical research.

\section{REFERENCE}

AlPERT, M. (1982). Encoding of feelings in voice. In P. J. Clayton \& J. E. Barrett (Eds.), Treatment of depression: Old controversies and new approaches (pp. 217-227). New York: Raven Press.

\section{ANNOUNCEMENT \\ Eleventh Annual Conference on Language Development \\ October 17, 18, and 19, 1986}

The Boston University School of Education invites papers for the 11th Annual Conference on Language Development, to be held on October 17, 18, and 19, 1986.

Papers on the topics of first and second language acquisition, bilingualism, language disorders, writing, literacy, narratives, ASL and sign language, neurolinguistics, sociolinguistics, theoretical language acquisition, and universal grammar will be considered for presentation at the conference. The deadline for submission of abstracts is June 1, 1986. This year's keynote speaker is Noam Chomsky from the Massachusetts Institute of Technology.

For more information write: Language Development Conference, School of Education, Boston University, Boston, MA 02215. 